\title{
Multidimensional Data Sets - Presentation, Evaluation and Extraction
}

\author{
M. Haschke ${ }^{1}$, U. Waldschläger ${ }^{1}$, R. Tagle ${ }^{1}$, U. Rossek $^{1}$ \\ ${ }^{1}$ Bruker Nano GmbH, Berlin, Germany
}

Micro-X-ray fluorescence spectrometry ( $\mu$-XRF) can measure element dependent intensity distributions. This creates multidimensional data sets which contain the intensities for different energies in dependence of the spatial coordinates. Elemental distributions give a good impression about the general structure of a sample and about the different composition of special objects in the investigated material. But these measurements can generate very large data sets - in dependence of the size of the analyzed area from several 100 MBytes to even considerably more than 1 GByte. This offers the question: How to handle these large data sets - for their effective presentation as well as for the extraction of the information of interest, for the objectification of the content or for the fast comparison with other distributions. This is a question which is relevant not only for elemental distributions measured by $\mu$ XRF but also for distributions of other parameters measured with other methods - for example phases measured with XRD, compounds measured with IR- or UV-spectroscopy or temporal changes of special parameters measured with in time resolved measurements.

One challenge for the data presentation is that only two dimensions are available for that purpose. With the help of different colors and variations of their brightness it is possible to visualize even higher dimensional data.

For the extraction of data a series of different methods are available. The first requirement is the collection of distributions and their processing that gives images of high quality. The collection of elemental distributions of high quality with $\mu$-XRF depends on the size of the analyzed area and the distance between the different areas but also on the amount of collected radiation. These distributions can be process by known procedures like averaging or smoothing to improve their quality. An important requirement for a good image quality is a high number of pixels to avoid pixilation.

Other possibilities are the use of image processing procedures for the improvement of the contrast between different components in the distribution, the evaluation of pixel data by deconvolution or quantification. But all these procedures still allow only the inspection of distributions and not yet the extraction of special parameters which characterize their composition.

There are very simple possibilities for an objectification like the display of intensity ratios between different elements. This would allow for example the normalization of matrix effects. This is interesting because the matrix influences the fluorescence intensity. Spatial as well as temporal changes of the matrix due to inhomogeneous sample composition or changes of the sample composition caused by external influences are often the reason for the analysis of materials with position sensitive or time resolved methods.

But the use of chemometric methods can be much more interesting. This can be for example the identification of pixels with similar composition and their presentation in the same way for example the display of phases in the same manner and the analysis of their distribution or concentration in the 
analyzed sample. This still allows only the inspection of distribution and not yet the extraction of their content.

For that purpose example methods of pattern recognition for the identification of special shapes of objects in the sample and their comparison with objects in other samples can be helpful.

So it can be concluded that the high amount of data in distribution should and can be reduced for a better understanding of the material. But the best procedure depends strongly on the actual requirements and on the structure of the analyzed material. An instrument should offer a wide range of possibilities but their selection depends on the analytical task.

Another interesting question is the quantification of distributions. Due to the very low intensities in every pixel the quantification procedure can fail. This can be avoided by a pixel binning which increase the pixel intensities but reduce the number of pixels i.e. the spatial resolution or the quality of distribution images. To find an objective compromise between spatial resolution and quality of quantification the data quality needs to be characterized - by their statistical distributions but also with the help by parameters like the limit of detection for structures in distributions. Despite of the very low number of counts as a result of extremely short measurement times per pixel elemental structures can be detected with a high sensitivity. The understanding of this effect and its use for the interpretation of data is important for a correct data evaluation.

Few of these questions will be discussed based on several data sets for elemental distributions that are collected with micro-X-Ray fluorescence. The analyzed samples are from geology and microelectronics. It will be demonstrated the influence of image processing to the image quality, the use of different methods for the objectification of the content of the distributions, the use of chemometric methods for the extraction of characterizing parameters of the distribution as well as the determination of general distribution parameters like limits of detection. 